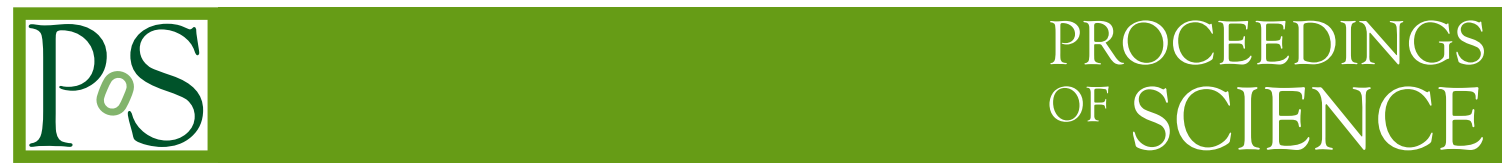

\title{
Overview of the EVN and its capabilities
}

\author{
T. Venturi ${ }^{* \dagger}$ \\ INAF, Istituto di Radioastronomia, Bologna, Italy \\ E-mail: tventurieira.inaf.it
}

I briefly review the main current features of the European VLBI Network and the way it operates. The $\mu \mathrm{Jy} /$ beam sensitivity of the array makes the EVN the ideal instrument for a wide range of radio astrophysical studies, from nearby galactic stellar objects to the most distant and youngest quasars and starburst galaxies. Moreover, rapid response science is a key feature of the EVN, thanks to the real-time observing capabilities at most EVN stations. The array is continuously evolving, in the effort to meet the needs of the scientific community. The roadmap for the future developments is given in the EVN2015 document, and the main steps are listed here.

The procedure to access EVN telescope time is described and relevant web links are given.

Potential new users of the EVN and e-VLBI networks may rely on the help and expertise of the Science Operation \& Support Group at JIVE.

10th European VLBI Network Symposium and EVN Users Meeting: VLBI and the new generation of radio arrays

September 20-24, 2010

Manchester Uk

\footnotetext{
* Speaker.

${ }^{\dagger}$ Chairperson of the EVN Program Committee at the time of the Symposium.
} 
Table 1: Radio Telescopes in the EVN

\begin{tabular}{lcrllrr}
\hline EVN Observatory & Telescope & $\mathrm{D}(\mathrm{m})$ & $\mid$ EVN Observatory & Telescope & $\mathrm{D}(\mathrm{m})$ \\
\hline Jodrell Bank (UK) & $\mathrm{Jb}-1$ (Lovell) & 76 & $\mid$ Seshan (Shanghai, CH) & $\mathrm{Sh}$ & 25 \\
& $\mathrm{Jb}-2(\mathrm{Mk} 2)$ & 25 & $\mid$ Nanshan (Urumqi, CH) & $\mathrm{Ur}$ & 25 \\
Cambridge (UK) & $\mathrm{Cm}$ & 32 & $\mid$ Torun (PL) & $\mathrm{Tr}$ & 32 \\
Westerbork (NL) & $\mathrm{Wb}$ Array & $\mathrm{N} \times 25$ & $\mid$ Metsahovi (FI) & $\mathrm{Mh}$ & 14 \\
Effelsberg (D) & $\mathrm{Eb}$ & 100 & $\mid$ Yebes (ES) & $\mathrm{Ys}$ & 40 \\
Medicina (I) & $\mathrm{Mc}$ & 32 & $\mid$ Arecibo (USA & $\mathrm{Ar}$ & 305 \\
Noto (I) & $\mathrm{Nt}$ & 32 & $\mid$ Hartebeesthoek (SA) & $\mathrm{Hh}$ & 26 \\
Onsala (S) & On-85 & 25 & $\mid$ Svetloe (RU) & $\mathrm{Sv}$ & 32 \\
& On-60 & 20 & $\mid$ Zelencgukskaya (RU) & $\mathrm{Zc}$ & 32 \\
Wetzell (D) & $\mathrm{Wz}$ & 20 & $\mid$ Badary (RU) & $\mathrm{Bd}$ & 32 \\
\hline
\end{tabular}

\section{The European VLBI Network}

The European VLBI Network (EVN) is a consortium of individual Institutes and Observatories, which share an overlapping plan of development and telescope observing time. The goal is to address milliarcsecond radio astrophysics, and the result of this combined effort is the largest and most sensitive very long baseline interferometry array in the world.

The EVN is actually much larger than Europe itself, going from Yebes (Spain) to Seshan (China) in East-West, and from Hartebeestoek (South Africa) to Onsala (Sweden) in North-South. The corresponding baselines are $9196 \mathrm{~km}$ in E-W and $8525 \mathrm{~km}$ in N-S. The maximum angular resolution of the array is in the range $\sim 0.15-5$ milliarcseconds, going from $43 \mathrm{GHz}$ to $1.6 \mathrm{GHz}$.

As of May 2011, the Consortium includes 12 countries, for a total of 20 radio observatories. Occasionally, and under certain conditions, more antennas can join the observing array. Figure 1 shows the distribution of the EVN radio telescopes around the world, and Table 1 reports the list of radio telescopes and their size.

The EVN Correlator at JIVE (Joint Institute for VLBI in Europe, Dwingeloo) is a fundamental constituent of the EVN. The majority of EVN-only and Global VLBI projects are correlated there (see Campbell, these proceedings, for an updated review of the correlator capabilities and future developments).

The EVN operates in 6 standard bands, from $1.6 \mathrm{GHz}$ to $22 \mathrm{GHz}$, including 6.0 and $6.7 \mathrm{GHz}$ ( $\mathrm{OH}$ and methanol bands respectively), and may occasionally observe at $327 \mathrm{MHz}$ and $43 \mathrm{GHz}$. Not all telescopes are equipped with the same receivers ${ }^{1}$, and the simultaneous array may include only a subset of antennas.

Table 1 clearly shows that the EVN has a very large collecting area, which combined with the routinely available $1 \mathrm{Gpbs}$ recording rate boosts the sensitivity of the array at the level of few $\mu \mathrm{Jy} / \mathrm{beam}$.

\footnotetext{
${ }^{1}$ The list of receivers available at each observatory, as well as their performances, can be found in the EVN status table at the address http://www.evlbi.org/user_guide/EVNstatus.txt.
} 


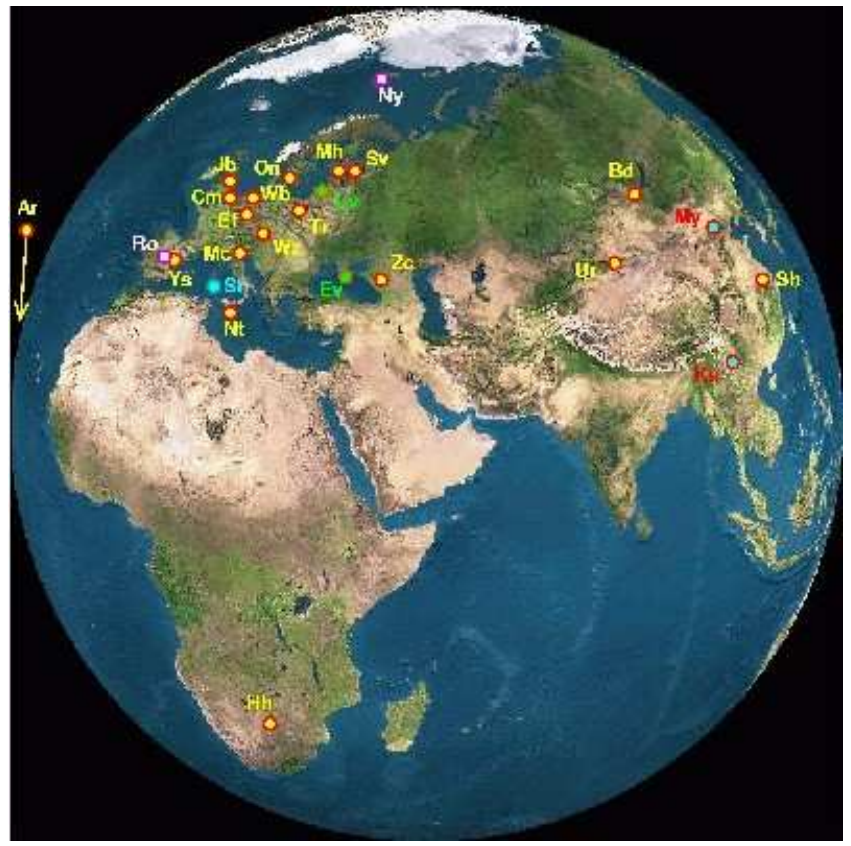

Figure 1: Colour-coded distribution of EVN telescopes around the world. Red/yellow=current operational EVN stations; cyan/red=existing telescopes likely to join the EVN in the near future; cyan/blue=new telescopes under construction; pink/purple=non-EVN stations which have occasionally participated in EVN observations; green/brown=non-EVN stations with whom initial EVN tests have been carried out for possible future inclusion in the array.

\section{An evolving array: real-time VLBI with the EVN}

Since 2006, the EVN is operating in real-time mode, thanks to the optical fiber link connection between the individual telescopes and the EVN correlator at JIVE. Thanks to the contribution of the EC through the EXPReS and NEXPReS projects [3], under FP6 and FP7 respectively, the development of e-VLBI has been very fast. An increasing number of EVN telescopes are part of the e-VLBI array, and we are now at a stage where the disk recording array and the real-time array are almost overlapping.

The relevance of the transition from standard disk recording to real-time VLBI is manyfold. First of all, real-time VLBI allows immediate access to the data. This is a major step forward if we think of the long timescales of disk-based recording VLBI, which opens up the door to rapid response science. Moreover, e-VLBI has turned out to be highly reliable, since failures or dropoffs can be taken care of immediately. Last but not least, it considerably improves the recording capacity of the array, now limited by disk space. The e-EVN programme is officially recognised as a SKA Pathfinder for its continuing contribution to the SKA's requirements for new methods of high rate data transport and processing.

The e-VLBI now operates at a sustainable recording rate of 1 Gbps at most stations ${ }^{2}$. In the spirit

\footnotetext{
${ }^{2}$ The e-EVN status table is available at http: / / www.evlbi.org/evlbi/e-vlbi_status.html. and is regularly updated.
} 


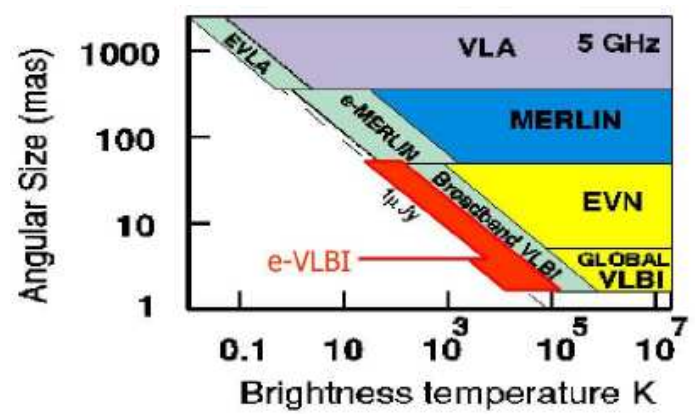

Figure 2: Sensitivity of the e-EVN compared to the new upgraded arcsecond scale arrays such as eMERLIN and EVLA. Courtesy of M. Garrett.

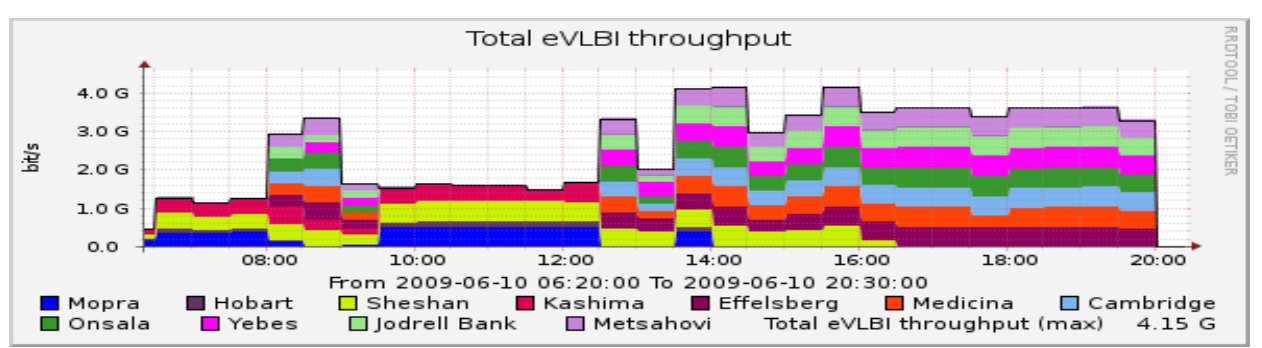

Figure 3: Data throughput of the first global e-VLBI experiment, carried out at $22 \mathrm{GHz}$ in May, June and July 2009, with the e-EVN and the Large Baseline Array (LBA, Australia) [2]. Courtesy of M. Giroletti.

of the EVN roadmap, as envisaged in the EVN2015 document ${ }^{3}, 4$ Gbps recording will soon be available at most stations, and the development to increase the recording capability up to $10 \mathrm{Gbps}$ is in progress. Figure 2 shows the sensitivity performance of the e-EVN compared to other modern upgraded arcsecond resolution arrays. An example of the e-VLBI data throughput for a successful global experiment is reported in Fig. 3: the sustainable data rate and the reliability of the observing run stand out clearly from the figure.

\section{EVN and other arrays}

The EVN may operate in coordination with other interferometers, such as the Very Long Baseline Array (VLBA), to perform Global VLBI observations, and the eMERLIN array (with a subset of the available receivers). A few EVN telescopes (Eb, On-60 and Mh) are equipped with high frequency receivers and participate in the Global mm-VLBI Array $\left(\mathrm{GMVA}^{4}\right)$ together with a subset of VLBA telescopes, Plateau de Bure and Pico Veleta (IRAM), expanding the EVN frequency coverage almost up to $100 \mathrm{GHz}$.

\section{Science with the EVN}

The present most outstanding features of the EVN are the large collecting area, the 1 Gbps recording capability and the real-time observing. Moreover, as of today, the EVN is the only VLBI

\footnotetext{
${ }^{3}$ available on web at the address http://www. evlbi.org/documents / .

${ }^{4}$ see GMVA web page at http://www.mpifr-bonn.mpg.de/div/vlbi/globalmm/.
} 
array which allows observations of the maser $\mathrm{OH}$ and methanol lines.

The sensitivity upgrade of the EVN over the past few years has opened up the door to the faint nearby and distant Universe, and nowadays a wide range of science can be addressed, from the various stages of stellar evolution, to the early days of the Universe ${ }^{5}$. Just to mention a few, very successful studies include the radio emission from local brown stars; the physics of Galactic star forming regions; the evolution of historical supernova explosions, such as SN 1993J; the structure of the Milky way through maser astrometry; nearby prolific star forming galaxies (such as Arp 220, M 82 and Arp 299); distant star forming galaxies and supermassive binary black holes; astrometric studies. The rapid response time of the e-EVN has turned out to be crucial for the study of galactic flaring objects, such as X-ray binary systems, for the participation in multiband observing runs of active galactic nuclei detected by the high energy observatories, such as Fermi, and for dense structural monitoring of galactic and extragalactic sources characterised by structural changes (one example is $\mathrm{M} 87$ ).

The papers in these proceedings cover all these fields and provide an excellent and complete overview of the current scientific potentials of the EVN. All the contributions presented at this conference are based on very recent projects and observations, and show that both data delivery and analysis are very fast and allow a timely publication of the results.

\section{Future developments}

The EVN is a continuously evolving array, in the effort to keep up with the demands and expectations of the radio astronomical community. The EVN2015 document provides the future actions for the development of the EVN and e-VLBI over the next few years, which can be briefly listed as follows:

- improvement of current performances: it includes large bandwidth receivers, higher data rate and frequency agility, to improve the sensitivity and flexibility of the array;

- development of new features: extension of the observing frequency range through a possible synergy with ALMA and LOFAR; inclusion of new telescopes in the array and development of a next generation correlator [1];

- easier access to the data.

\section{How to access the EVN}

The EVN offers three disk-based recording observing sessions each year, the so-called "standard sessions", and 10 e-VLBI runs. At present the e-VLBI runs last 24 hours and only one frequency/run is offered. Proposals to access EVN, global VLBI and e-VLBI time may be electronically submitted three times/year, with deadlines on $1^{\text {st }}$ February, $1^{\text {st }}$ June and $1^{\text {st }}$ October each year 6 .

\footnotetext{
${ }^{5}$ The linear resolution of the EVN ranges from the order of magnitude of the astronomical unit $(10$ mas=50 AU at the distance of SS 433) to that of the parsec ( 1 mas $\sim 6 \mathrm{pc}$ at $\mathrm{z}=5.8$ ).

${ }^{6}$ General information and guidelines for proposal submission may be found at the web address http://www.ira.inaf.it/evn_doc/guidelines.html.
} 
A catalogue of all sources observed with the EVN, starting from 1990, is accessible on web ${ }^{7}$, and cross-linked with the EVN Archive at JIVE $^{8}$, which provides also FITS files and other plots, according to the EVN data access policy.

In the effort to help the VLBI users' community and to make the EVN and e-VLBI an accessible and users' friendly facility, each experiment correlated at the EVN correlator at JIVE is preliminary reduced by the JIVE support staff members through a pipeline. The final products delivered to the users are the raw correlated data and the outcome of the preliminary data reduction.

New (but also experienced) users may receive help and assistance by the Science Operation \& Support Group at JIVE, from technical support during the proposal preparation, to the data reduction. Visits to JIVE are encouraged, and support is provided by the EC through the TransNational Access Program under the Seventh Framework Programme (FP7-RadioNet) ${ }^{9}$.

\section{Acknowledgments}

The European VLBI Network is a joint facility of European, Chinese, South African and other radio astronomy institutes funded by their national research councils. This activity is supported by the European Community (EC) through the Framework Programme FP7, Advanced Radio Astronomy in Europe, grant agreement No. 227290. The Joint Institute for VLBI in Europe provides support to the world-wide EVN user community and supports the EVN operations.

\section{References}

[1] Campbell, R.M., 2011, these proceedings

[2] Giroletti, M., et al., 2011, A\&A, 528, L11

[3] van Langevelde, H., 2010, ISKAF2010 Science Meeting, Proceedings of Science, arXiv:1009:2911

\footnotetext{
${ }^{7}$ http://db.ira.inaf.it/evn/.

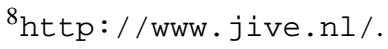

${ }^{9}$ Information may be found at the web address: http://www. jive.nl/evn-travel-support-data-analysis.
} 